\title{
WARM DARK MATTER MODEL OF GALAXY FORMATION
}

\author{
Y.P. JING \\ Shanghai Astronomical Observatory, Partner Group of MPI für Astrophysik, Nandan \\ RD 80, Shanghai 200030, China \\ E-mail:ypjing@center.shao.ac.cn
}

\begin{abstract}
Cold Dark Matter (CDM) models of galaxy formation had been remarkably successful to explain a number of observations in the past decade. However, with both the theoretical modeling and the observations being improved, CDM models have been very recently shown to have excessive clustering on the sub-galactic scale. Here I discuss a solution, based on our high-resolution numerical simulations, to this outstanding problem by considering Warm Dark Matter (WDM). Our results show that the over-clustering problem on sub-galactic scales can be overcome by WDM models, and all the advantages of CDM models are preserved by WDM models. Therefore, the WDM model will become an interesting alternative to the well-studied CDM models
\end{abstract}

\section{Introduction}

Cold Dark Matter (CDM) models have been shown very successful to explain many observations of galaxies on scales of about one $h^{-1} \mathrm{Mpc}$ to a few hundred $h^{-1} \mathrm{Mpc}$. But such models probably predict over-clustering on smaller scales, as recent highresolution simulations (Moore et al. 1999, Klypin et al. 1999, Jing \& Suto 2000) have shown. The halo density profiles in these simulations are steeper than those inferred from the rotation curves of low surface brightness (LSB) galaxies, and there are too many sub-halos within galactic halos when compared to the observed number of satellite galaxies around the Milky Way. There is also additional evidence for such overclustering from, e.g. the luminosity function of dwarf galaxies. Although some of these discrepancies may be resolved by introducing additional astrophysical processes (Bullock et al. 2000) and some others by properly interpreting the observations (van den Bosch \& Swaters 2000), there are attempts to resolve the discrepancies by revisiting the assumption about the dark matter (DM). A list of the candidates for replacing CDM proposed since the summer of 1999 includes selfinteracting DM, warm dark matter (WDM), repulsive DM, fuzzy DM, annihilating DM etc (see Davè et al. 2000 for references). In this talk, I will present an extensive study for a warm dark matter model using high resolution N-body simulations. Our results will show that the WDM model is in good agreement with the observational data without resorting to not-well-understood astrophysical processes. A complete description of the study appeared in our recent paper submitted to the Astrophyical Journal (Jing 2000).

\section{Model and Simulations}

We consider a model dominated by WDM with the matter density $\Omega_{0}=0.3$, the cosmological constant $\lambda_{0}=0.7$, and the Hubble constant $H_{0}=100 \mathrm{~h}=$ $67 \mathrm{kms}^{-1} \mathrm{Mpc}^{-1}$. The primordial power spectrum is $\propto k$, and the transfer function is taken from Bardeen et al. (1986) with a zero baryon content. The free-streaming

ms: submitted to World Scientific on November 5, 2018 
cutoff parameter $R_{f}=0.1 h^{-1} \mathrm{Mpc}$ is adopted, which is also consistent with the Ly- $\alpha$ forest observations (Narayanan et al. 2000). The linear power spectrum is normalized so that the current rms linear density perturbation within a sphere of radius $8 h^{-1} \mathrm{Mpc}$ is 1 . Because all the parameters except $R_{f}$ have usually been assumed for the low-density flat CDM (LCDM) model which best fits observations on scales of $1 h^{-1} \mathrm{Mpc}$ and up, this WDM is expected to fit these observations as well since the free-steaming of the warm dark matter has little effect on these scales. Thus our study will focus on the properties on galactic and sub-galactic scales, where the free-streaming effect of the WDM is expected to become significant. To single out the free-streaming effect, we compare the results of the WDM to those of the LCDM, which in most cases can effectively eliminate the numerical artifacts.

We have run a large set of cosmological simulations with box sizes of $12.5 h^{-1} \mathrm{Mpc}, 25 h^{-1} \mathrm{Mpc}$ and $50 h^{-1} \mathrm{Mpc}$ respectively. For each box size, three realizations are produced and $128^{3}$ particles are adopted for each model. The same initial phases are used for the WDM model and for the LCDM model. We have selected five WDM halos of $\sim 1000$ particles for each boxsize as well as the corresponding halos in the LCDM simulations. The virial mass of these halos is $7 \times 10^{10} M_{\odot}, 6 \times 10^{11} M_{\odot}$, and $5 \times 10^{12} M_{\odot}$ respectively from the small to the large boxsizes. We then use the Nested-Grid-P ${ }^{3} \mathrm{M}$ (Jing \& Suto 2000) to simulate these halos with a much higher resolution. A total of $\sim 7 \times 10^{5}$ particles are used for simulating each halo, with $\sim 5 \times 10^{5}$ particles (small) for the high-resolution region and $\sim 2 \times 10^{5}$ (massive) for the coarse-resolution region. About $3 \times 10^{5}$ particles from the high-resolution region will end up in the virialized region of the halo. Using the coarse (massive) particles can properly account for the tidal force which is important for forming the internal structures of the halos. A detailed account about the simulation technique can be found in Jing \& Suto (2000).

\section{Results}

We have made a very detailed analysis both for the cosmological simulations and for the high-resolution halo simulations. These results were presented in the talk, but can not be accommodated in this proceedings paper because of the limited space. A detailed account of these results can be found in our journal paper (Jing 2000). Here we just highlight a few interesting results.

In Figure 1 we show the differential mass function of dark matter halos in the WDM model as well as in the LCDM model at redshift $z=4$. The mass function is defined as the mean number density of halos within a unit logarithmic interval of halo mass. Because of the free-streaming motion of the warm dark matter, the halo abundance in the WDM model is smaller than in the LCDM model at subgalactic scales. The WDM halos are about 2 times and 4 times less abundant at $M=10^{11} M_{\odot}$ and $M=2 \times 10^{10} M_{\odot}$ respectively. A crucial test for this effect would be the hydrogen content of the damped Ly- $\alpha$ systems. Since the halo density is reduced only by a factor of two on the relevant scales, the WDM is well consistent with the observations of the damped Ly- $\alpha$ systems (see Mo \& Miralda-Escude 1994, Ma et al. 1997)

The density profiles of dark matter halos are presented in Figure 2. We found

ms: submitted to World Scientific on November 5, 2018 


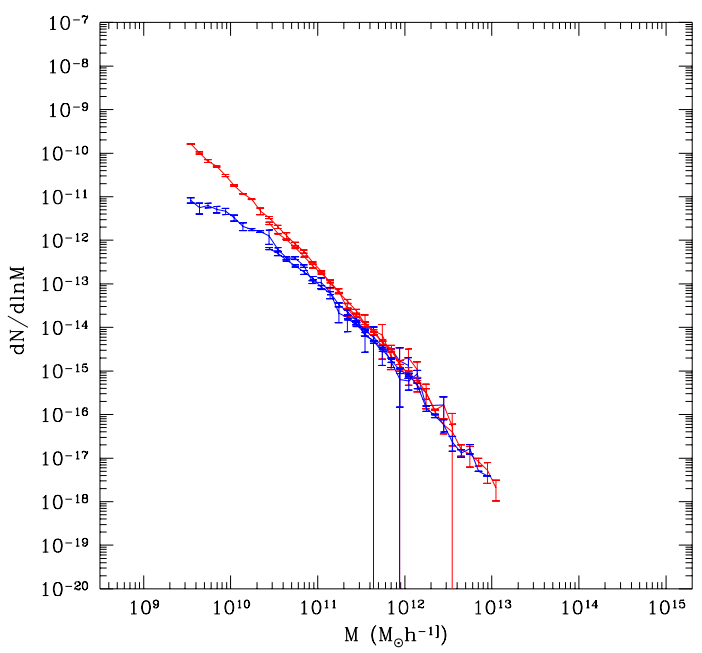

Figure 1. The differential mass function of dark matter halos at redshift $z=4$ in the WDM model (lower) and in the LCDM model (upper).

that the profiles of the halos less massive than $5 \times 10^{11} M_{\odot}$ are significantly flatter in the WDM model. Especially the halos with the mass similar to the those of the dwarf galaxies have formed cores at their centers, which could be potentially important for explaining the slowly rising rotation curves observed for the LSB galaxies. Our comparison of the halo circular velocity with the observed rotation curves shows that the LCDM model could be ruled out for its too steep density profiles, and the WDM model is consistent with the observations of LSB galaxies.

Now let's consider sub-halos within virialized halos. The smoothed density of each dark matter particle is estimated in the way used in Smoothed Particle Hydrodynamics simulations. The average of the smoothed density is calculated for each radial shell, and the particles with the smoothed density five times above the radially averaged value are identified. These identified particles are grouped with the friend-of-friend algorithm of a bonding length equal to one tenth of the global mean particle separation. We find that the resulted catalogue of the sub-halos is quite robust against the parameters we have taken. Figure 3 shows the number of sub-halos with the circular velocity larger than $v_{c}$. There are much fewer sub-halos in the WDM halos than in the LCDM halos. To compare with obsrevations, we plot the observed abundance of the satellite galaxies within the Milky Way which has a circular velocity of $220 \mathrm{~km} \mathrm{~s}^{-1}$. The number of sub-halos within a Milky Way like halo, which should be in between the curves of $v_{c, \text { host }}=270 \mathrm{~km} \mathrm{~s}^{-1}$ and of $v_{c, \text { host }}=135 \mathrm{~km} \mathrm{~s}^{-1}$, can be readily read out for the two dark matter models. The LCDM halos have too many halos as many previous studies have pointed out, but the WDM halos have a comfortable amount of sub-halos which is in good agreement

ms: submitted to World Scientific on November 5, 2018 


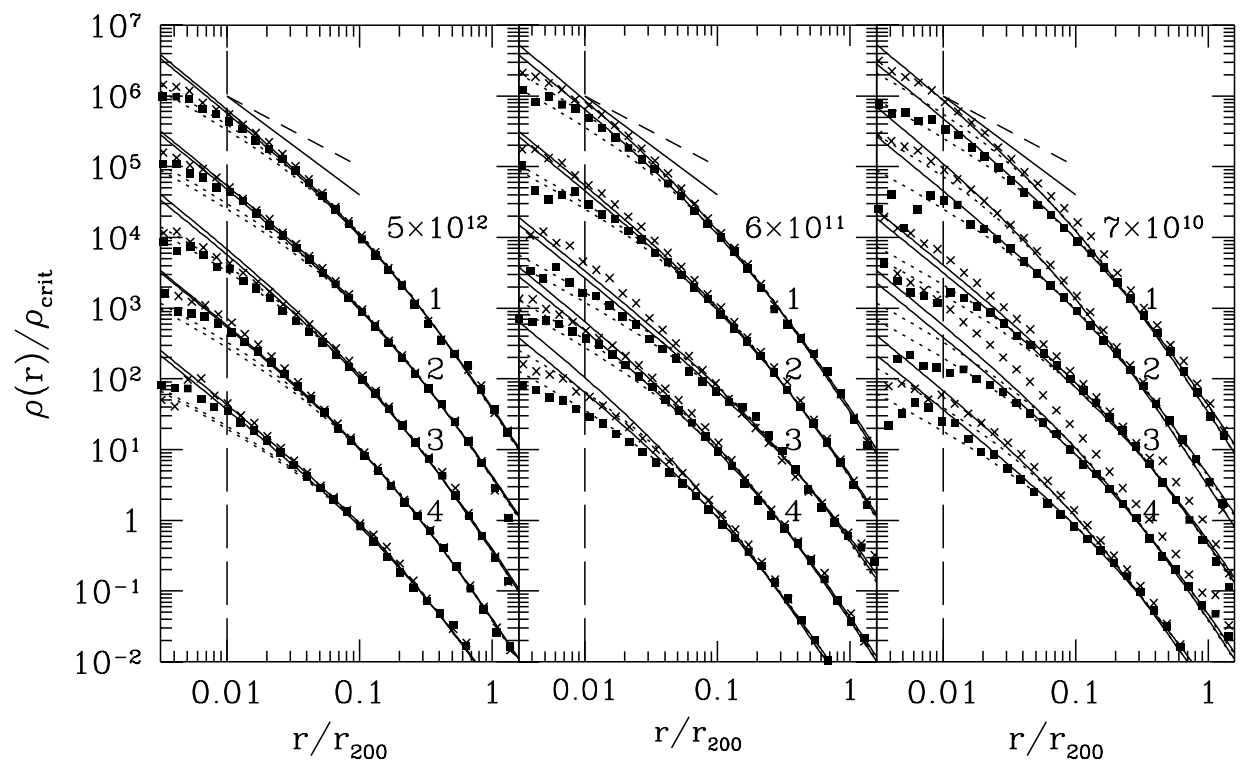

Figure 2. The density profiles of the dark matter halos in the WDM model (squares) and in the LCDM model (crosses).

with the observations. We noted that the our results for the LCDM are in good agreement, for the mass range plotted in the figure, with the result of Moore et al.(1999).

In summary, we have presented a first, very detailed simulation study for the WDM model. Our results show that the model predicts enough halos at high redshift $z \approx 4$ to be consistent with the observations of damped Ly- $\alpha$ systems. This model is also consistent with the clustering of the Ly- $\alpha$ absorption lines (Narayanan et al. 2000). The density profiles of the halos are significantly flatter than in the LCDM model for halo mass less than $\sim 10^{11} M_{\odot}$, bringing about good agreement with the recent high-resolution observation of the rotation curves of LSB galaxies by Swaters et al.(2000). In contrast, we found that the CDM halos are NOT consistent with the observation of Swaters et al.. There are significantly fewer subhalos in WDM halos than in LCDM halos, and the number of subhalos within Milky Way like halos in the WDM model agrees very well with the observed number of satellite galaxies around the Milky Way without resorting to poorly-understood astrophysical processes. Since there are much fewer sub-halos, we expect that the over-cooling problem on the galactic scales can be alleviated and large galactic disks can be formed, which has been a serious difficulty for CDM models. All these attractive features of the WDM model warrant further detailed studies of this model.

The work is supported by the One-Hundred-Talent Program and by nkbrsfg19990754.

ms: submitted to World Scientific on November 5, 2018 


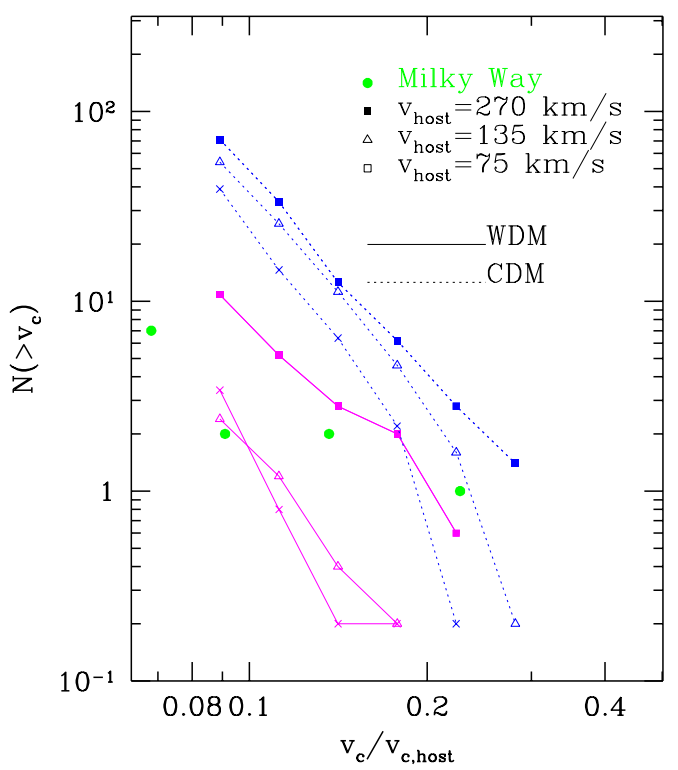

Figure 3. The number of sub-halos with the circular velocity larger than $v_{c}$ as a function of $v_{c} / v_{c, h o s t}$ where $v_{c, h o s t}$ is the circular velocity of the virialized host halo where the sub-halos reside.

\section{References}

1. Bullock, J. S., Kravtsov, A. V., \& Weinberg, D. H, 2000, ApJ, submitted (astro-ph/0002214)

2. Davé, R., Spergel, D.N., Steinhardt, P.J., Wandelt, B.D., 2000, ApJ, submitted (astro-ph/0006218)

3. van den Bosch, F. C., Swaters, R. A. 2000, AJ, submitted, astro-ph/0006048

4. Jing, Y. P. \& Suto, Y. 2000, ApJ, 529, L69

5. Jing, Y. P. 2000, ApJ, (submitted)

6. Klypin, A., Kravtsov, A. V., Valenzuela, O., \& Prada, F., 1999, ApJ, 522, 82

7. Ma, C., Bertschinger, E., Hernquist, L., Weinberg, D. H. \& Katz, N. 1997, ApJ, 484, L1

8. Swaters, R. A., Madore, B. F. \& Trewhella, M. 2000, ApJ, 531, L107

9. Mo, H. J. \& Miralda-Escude, J. 1994, ApJ, 430, L25

10. Moore, B., Ghigna, S., Governato, F., Lake, G., Quinn, T., Stadel, J., \& Tozzi, P., 1999, ApJ, 524, L19

11. Narayanan, V., Spergel, D. N., Davé, R. \& Ma, C.-P. 2000, ApJ, submitted

ms: submitted to World Scientific on November 5, 2018 